\title{
Comparative transcriptomic studies identify specific expression patterns of virulence factors under the control of the master regulator PhcA in the Ralstonia solanacearum species complex
}

\author{
Anthony Perrier, Xavier Barlet, Rémi Peyraud, David Rengel, Alice Guidot*, Stéphane Genin** \\ LIPM, Université de Toulouse, INRA, CNRS, Castanet-Tolosan, France
}

\section{A R T I C L E I N F O}

\section{Keywords:}

Plant pathogen

Regulation

RNA-seq

Type 3 secretion system

Quorum sensing

Bacterial wilt

\begin{abstract}
A B S T R A C T
The global regulator PhcA controls numerous traits associated to virulence and bacterial proliferation in strains of the plant pathogen Ralstonia solanacearum species complex. Here, we conducted a genome-wide RNA sequencing study of the GMI1000 wild-type strain and a derived phcA mutant grown in complete medium. The PhcA regulon we identified is the largest regulon described to date in the $R$. solanacearum species complex with 1581 regulated genes, representing about 30\% of the bacterial genome. Among these genes, 166 transcription regulators were identified including known regulators controlling major cellular functions such as the Type 3 secretion system and 27 novel regulators that were not identified in previous transcriptomic studies. This study highlights that PhcA controls other functions beside pathogenicity stricto sensu which participate to the global cell homeostasis (metabolism, energy storage). We then compared the PhcA regulon identified in complete medium to the recently published PhcA regulon obtained in planta. This comparison of the set of GMI1000 genes subjected to PhcA regulation in both conditions revealed 383 common genes. Among them, 326 (85\%) had a similar PhcA dependent regulation pattern in complete medium and in planta, and 57 (15\%) displayed an opposite regulation pattern. A large majority of the genes repressed by PhcA in complete medium but activated in planta belong to the HrpG-HrpB regulon, which represents a set of key genes required for $R$. solanacearum pathogenesis. This latter class of genes appears to be specifically induced by PhcA in the plant environment whereas PhcA represses their expression in complete medium. The large set of direct and indirect targets identified in this study will contribute to enrich our knowledge of the intricate regulatory network coordinating the expression of virulence and metabolic functions in the model plant pathogen $R$. solanacearum.
\end{abstract}

\section{Introduction}

Strains from the Ralstonia solanacearum species complex (RSSC) cause wilt disease of more than 250 plant species distributed around the world, mainly under tropical climates. Bacteria enter susceptible plants through the roots, invade the xylem vessels and spread rapidly to aerial parts of the plants. Extensive colonization of the vascular system and production of large amounts of exopolysaccharides (EPS) alter water fluxes in the plant, causing wilting symptoms and eventual plant death.

RSSC pathogenesis is regulated by a complex regulatory network that responds to the soil and plant environments, the presence of plant cells and the bacterial cell density [1]. At the center of this network is the global regulator PhcA, a LysR-type transcriptional regulator that controls directly or indirectly expression of many virulence factors such as EPS production, plant cell wall degrading enzymes, bacterial motility and the Type 3 secretion system (T3SS) that allows the translocation of effector proteins into plant cells $[2,3]$.

Levels of functional PhcA are regulated in response to bacterial cell density by a quorum-sensing (QS) mechanism that involves methyl 3hydroxypalmitate (3-OH PAME) or methyl 3-hydroxymyristate (3-OH MAME) as a QS signal $[4,5]$. Therefore, at low cell density, for example during saprophytic life in soil or during early plant colonization steps, levels of functional PhcA are low, leading to a low virulence phenotype and expression of both twitching and swimming motility optimized for survival in soil or water, and invasion of plant tissues. In contrast, at high cell density, such as during plant xylem colonization, the accumulation of the QS signal leads to production of abundant functional PhcA and, subsequently, production of multiple virulence factors while suppressing production of survival/invasion factors. Recently, Peyraud et al. [6] reported that PhcA controls a trade-off between virulence

\footnotetext{
* Corresponding author.

** Corresponding author.

E-mail addresses: alice.guidot@inra.fr (A. Guidot), stephane.genin@inra.fr (S. Genin).
} 


\author{
Abbreviations \\ FDR False Discovery Rate \\ RNA-seq RNA sequencing \\ WT wild-type \\ DEGs Differentially Expressed Genes
}

T3E

T3SS

T6SS

QS

HSL

RSSC
Type 3 Effector

Type 3 Secretion System

Type 6 Secretion System

Quorum sensing

Homo-Serine Lactone

Ralstonia solanacearum species complex factor production and bacterial proliferation in a RSSC strain. Indeed, a phcA mutant is avirulent but, as a consequence of not paying the cost for virulence, has a better growth rate and is able to metabolize 17 substrates more than the wild-type strain [6,7].

Genetic studies identified numerous traits known to be positively or negatively regulated by PhcA [1,2]. However, the whole PhcA regulon was only characterized recently through global transcriptome studies in strains GMI1000 and OE1-1 (a strain phylogenetically close to GMI1000) [7,8]. These genome-wide RNA sequencing (RNA-seq) studies were conducted using either total RNA extracted during tomato colonization (strain GMI1000) [7] or after growth in minimal medium (strain OE1-1) [8]. Here, we conducted a RNA-seq profiling study for the GMI1000 wild-type strain and a derived phcA mutant grown in complete medium. In order to evaluate the relevance of plant-derived signals in the activation of virulence determinants, we also compared the PhcA regulon in complete medium to the recently published PhcA regulon obtained in planta. Our analysis revealed that PhcA controls an extremely large set of genes corresponding to biological functions that extend beyond pathogenicity in the strictest sense. It also pinpoints a class of pathogenicity factors (including T3SS) which are subjected to a specific PhcA dependent regulation pattern, being repressed when bacteria are grown in complete medium but induced once inside the plant.

\section{Material and methods}

\subsection{Bacterial strains and growth conditions}

R. pseudosolanacearum strains used in this study are the wild-type (WT) strain GMI1000 [9] and the phcA disruption mutant derivative

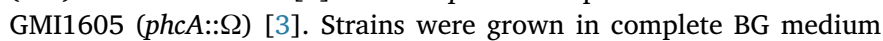
[10]. For agar plates, BG medium was supplemented with D-Glucose (5 g liter-1) and triphenyl-tetrazolium chloride (0.05 g liter-1). When needed, spectinomycin was added to the media at a final concentration of $40 \mathrm{mg}$ liter-1.

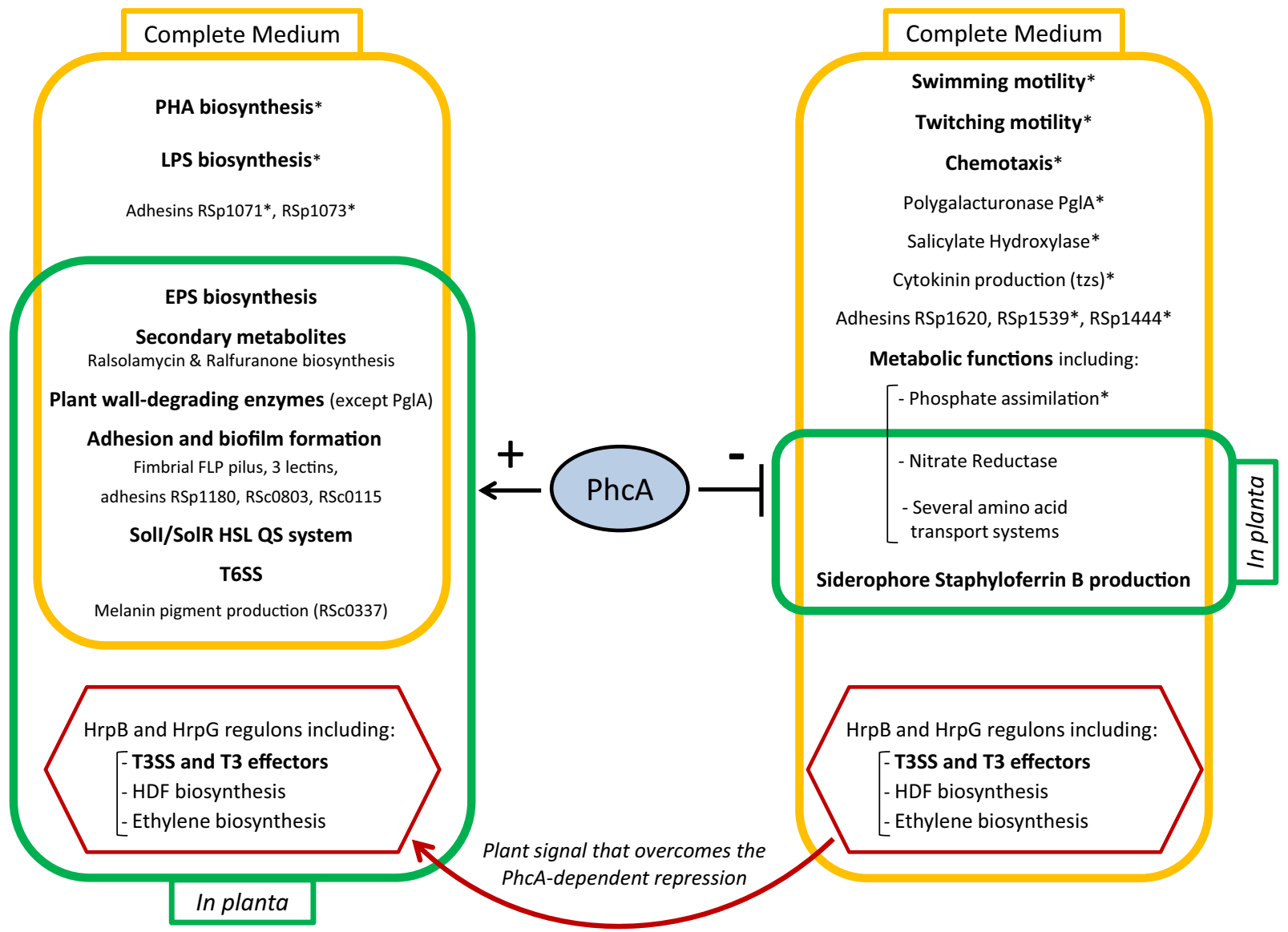

Fig. 1. Overview of the $R$. solanacearum traits regulated by PhcA based on transcriptomic analyses.

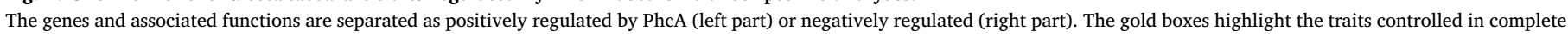

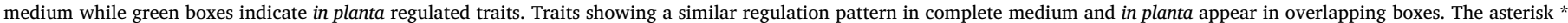

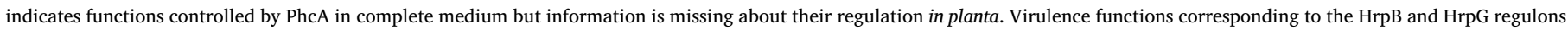

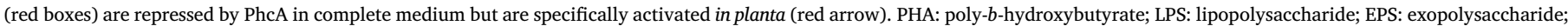

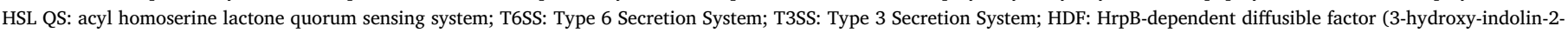
one). (For interpretation of the references to colour in this figure legend, the reader is referred to the Web version of this article.) 


\subsection{RNA extraction, depletion of rRNA and sequencing}

Total RNA was extracted from the wild-type (WT) GMI1000 strain and the phcA:: $\Omega$ mutant strain growing in BG medium at comparable cell densities $\left(\mathrm{OD}_{600 \mathrm{~nm}}-0.5\right)$. Three biologically independent experiments were conducted for each strain. Before RNA extraction, the bacterial culture was first stopped by mixing $1 \mathrm{ml}$ ethanol/phenol (95:5) to $20 \mathrm{ml}$ of culture during $3 \mathrm{~min}$ using a vortex. The culture was then centrifuged at $4000 \times g$ for $10 \mathrm{~min}$ at $4{ }^{\circ} \mathrm{C}$ and the pellet was resuspended in $200 \mu \mathrm{H} 2 \mathrm{O}$ RNase free for total RNA extraction. Total RNA was isolated and depleted of ribosomal RNAs as previously described [11].

Oriented paired-end RNA sequencing ( $2 \times 125 \mathrm{bp}$ ) was carried out by Fasteris (Fasteris SA, Plan-les-Ouates, Switzerland), using an Illumina HiSeq 2500 instrument and the procedures recommended by Illumina, with adaptors and amplification primers designed by Fasteris. The size of selected inserts was 150-250 bp. Libraries were sequenced in pairedend.

\subsection{Mapping and statistical analysis of RNAseq data}

Read pairs were mapped using the glint software (http://lipmbioinfo.toulouse.inra.fr/download/glint/) with parameters set as follows: matches $\geq 40$ nucleotides, with $\leq 3$ mismatches, only best-scoring hits taken into account. Ambiguous matches (same best score for several read-pairs) were removed. Finally, between 16.8 and $29.6 \mathrm{M}$ non ambiguous read-pairs were obtained. Mapped reads were imported into $\mathrm{R}$ environment. The package HTSFilter was used to eliminate very lowexpressed genes from the analysis. A total of 4990 genes out the 5307 predicted genes were thus kept in. R package DESeq2 was used to normalize and complete the differential analysis by conducting the built-in Wald test [12]. The $p$-values thus obtained were adjusted for multiple comparisons using the false-discovery rate (FDR) method [13]. Genes with a FDR-adjusted $p$-value (padj,FDR) $<0.005$ and an absolute Fold Change $|\mathrm{FC}|>2$ between strains were taken into further consideration in this work.

\section{Results and discussion}

\subsection{The PhcA regulon is wider in complete medium than in planta}

We analyzed the in vitro transcriptome of the GMI1000 and the $p h c A:: \Omega$ strains grown in complete medium. Differentially expressed genes (DEGs) ( $>2$-fold differentially expressed; $p$-value (padj,FDR) $<0.005$ ) between the two strains confirmed that PhcA is a major master regulator since the comparative analysis identified 1581 DEGs, including 787 up-regulated and 794 down-regulated genes, representing about $30 \%$ of the GMI1000 strain coding sequences (Table $\mathrm{S} 1)$. The PhcA regulon is therefore the largest regulon described to date in the $R$. solanacearum species complex.

The central position of PhcA in the RSSC regulatory network is illustrated by the fact that 166 genes annotated as transcription regulators are under the direct or indirect control of PhcA in complete medium (positive regulation for 42 transcription regulators, negative regulation for 122 transcription regulators). Many of these target transcription regulator genes are themselves known regulators of major cellular functions such as $h r p B / h r p G$ (T3SS and other pathogenicity functions), oxyR (antioxidant response), flhC (flagellar motility), cheY (chemotaxis), ntrC and phoB (nitrogen and phosphate assimilation, respectively), epsR and $x p s R$ (EPS biosynthesis) or solR (acyl-HSL responsive regulon) (Table S1) [1,2]. In agreement with previous studies $[7,8]$, the cellular processes regulated by PhcA in complete medium also include Type 6-dependent secretion system (T6SS), twitching motility, siderophore production, adhesins and lectins presumably involved in biofilm formation. Among the PhcA regulated traits identified in our study are chemotaxis, Flp pili required for biofilm formation
[14], some plant cell wall degrading enzymes (pglA, pehC and pme), biosynthesis of the core lipopolysaccharide [15], poly- $\beta$-hydroxybutyrate storage genes [16], the tzs gene (cytokinin biosynthesis) or the secondary metabolites ralstonins/ralsolamycin A \& B $[17,18]$ (Fig. 1).

In addition, we identified novel target genes negatively regulated by PhcA that encode for metabolite transporters such as phosphate utilization genes (RSc1529-RSc1535) and several ABC transport systems of unknown function (e.g. RSc1791-RSc1794, RSc2039-RSc2040, RSc3407-RSc3410, RSp1447). On the 199 genes with measured transcripts and annotated to be part of an ABC transporters, we found only 15 to be negatively regulated by PhcA in complete medium. These observations further support the recent finding that the enlarged metabolic versatility of a $p h c A$ mutant compared to the wild-type strain at high cell density is due to a resource allocation trade-off between growth and virulence instead of a transcriptional control of substrate usage [6]. The RSc0337 gene encoding a tyrosine hydroxylase responsible for the melanin pigment produced by GMI1000 [19] was found to be positively regulated by PhcA, in agreement with the observed phenotype of pigment production at high cell density when the strain is grown on complete medium plate. Finally, our analysis unraveled 27 novel target genes coding for transcription regulators that were positively regulated by PhcA in complete medium and were not identified in previous transcriptomic studies.

The recently published in planta transcriptome of the GMI1000 and phcA mutant strains revealed a total of 620 DEGs ( $>2$-fold differentially expressed; $p$-value (padj,FDR) $\leq 0.005$ ) [7]. Using the same cutoff values, the number of DEGs in complete medium was more than twice the number of DEGs obtained in planta. It is worth mentioning that many genes or functions, such as flagellar and twitching motility or metabolic genes known to be regulated by PhcA according to phenotypic analyses $[1,6,20,21]$, were present in the DEGs from the in vitro transcriptome studies performed in complete and minimal medium [8] but absent in the DEGs from the in planta transcriptome study [7]. Surprisingly, several genes previously described as PhcA-regulated and expressed in planta (e.g. hrpG, vsrC, numerous T3E and T3SS genes) [2] were also absent in the DEGs from the in planta transcriptome study [7]. This discrepancy could be the consequence of a technical bias either in the RNA sampling or RNA sequencing, thus leading to an under-representation of expressed genes in planta. If this is the case, it is therefore likely that some of the novel PhcA regulated genes identified in our study are also controlled in a similar fashion under in planta conditions.

\subsection{The PhcA-dependent regulation pattern in rich medium is mostly conserved in planta}

In order to compare the set of GMI1000 genes subjected to PhcA regulation in complete medium and in planta, we took the list of 787 and 794 genes up-regulated and down-regulated, respectively, in the phcA mutant in complete medium and looked for their expression pattern in planta using the Khokhani et al. dataset [7]. First, we observed that a large majority of the PhcA regulated genes in complete medium could not be compared (687 repressed and 511 activated genes) since these genes were absent (i.e no value provided) in the Khokhani et al. [7] dataset (Fig. 2). The PhcA dependent genes expressed in both conditions comprised 383 genes with 100 genes upregulated and 283 genes down-regulated in complete medium (Fig. 2). Among these 383 genes, $326(70+256)$, i.e $85 \%$, had a similar PhcA dependent regulation pattern in complete medium and in planta. This set of genes included the two previously described direct targets of PhcA, XpsR [22] and PrhIR [23], which are respectively activated and repressed by PhcA. It also included many other virulence-related genes positively regulated by PhcA in both environmental conditions such as EPS (epsABCDEFPR), lectins ( $r s l, r s l 2$ and RSp0569), glucanases (eglA, cbhA), T6SS (tssABDEHJKL), adhesins (RSc0115, RSp1180, RSp1605), 


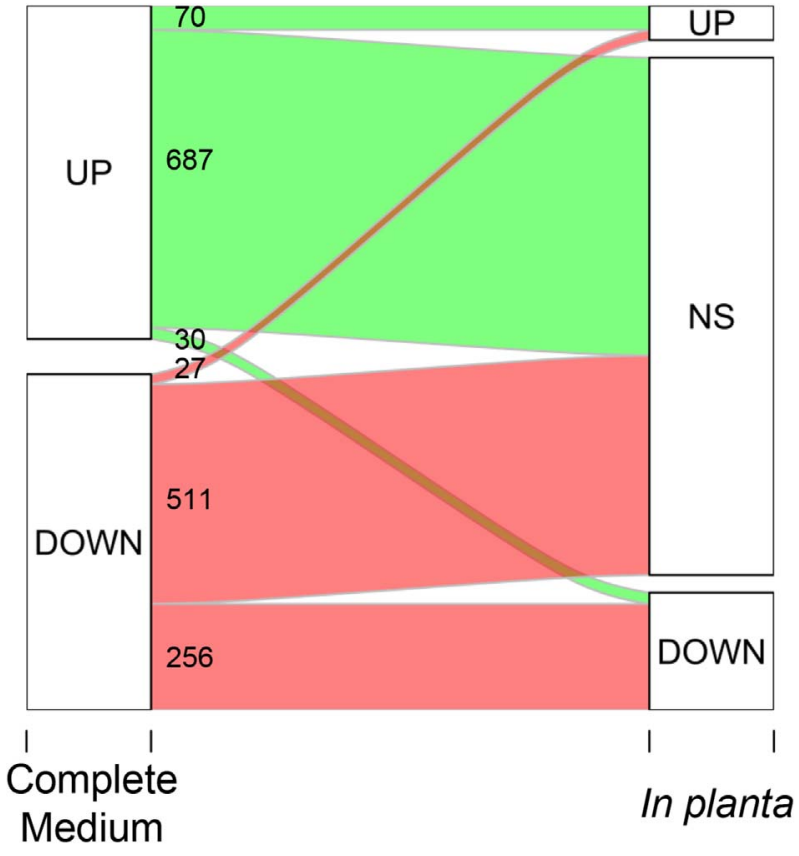

Fig. 2. Alluvial plot showing the distribution of PhcA-dependent genes in planta among the PhcA-dependent genes in complete medium.

In a $R$. solanacearum GMI1000 phcA mutant strain, (i) 70 up-regulated genes in complete medium are also up-regulated in planta, while 30 genes are down-regulated and (ii) 256 down-regulated genes in complete medium are also down-regulated in planta, while 27 are up-regulated. A large set of genes $(687+511)$ is annotated NS in planta, indicating that they are either not differentially expressed or absent from the in planta dataset [7].

ralfuranone biosynthesis (ralA and $\mathrm{ralD}$ ) and the acyl-homoserine lactone quorum sensing transcriptional activator (solR). On the other hand, the nitrate reductase (narGHJI) and siderophore biosynthesis genes (RSp0416-RSp0424) were found to be negatively regulated by PhcA in both conditions.
3.3. A subclass of pathogenicity genes is specifically activated by a signal perceived in plant that overcomes the PhcA-dependent repression

Among the 383 PhcA dependent genes that were differentially expressed both in complete medium and in planta, $57(30+27)$ genes (15\%) displayed an opposite PhcA regulation pattern (Fig. 2). In the phcA mutant, 30 genes were down-regulated in planta but up-regulated in complete medium (Fig. 2, Table 1) and 27 genes were up-regulated in planta but down-regulated in complete medium (Fig. 2, Table 2). The 27 genes up-regulated in planta and down-regulated in complete medium comprised a majority of $\mathrm{ABC}$-type transporters for unidentified metabolites, except for one annotated as sugar transporter (RSc0547) (Table 2). This class of transporters specifically induced in planta in a $p h c A$ mutant highlights a probable specific metabolic adaptation of the $p h c A$ mutant in the plant xylem environment.

Among the $30 \mathrm{PhcA}$ regulated genes that were up-regulated in rich medium but down-regulated in planta (Fig. 2), seven genes were also down-regulated in a phcA mutant grown in minimal medium conditions [8], thus suggesting that regulation for these genes is not plant-specific. Among the 23 remaining genes, 16 genes were reported to be HrpBdependent $[24,25]$ and included five T3E genes (ripAB, ripX, ripAD, ripF1_1 and ripAC), two structural genes of the T3SS ( $h r p Y$ and $h r c C$ ), three genes belonging to the $h d f$ locus ( $h d f A, h d f B, h d f F$ [26]) and other hrpB-regulated genes (RSc0616, RSc0617, RSp0840, RSp0882, RSp1461) [25] (Table 1). The last seven genes included four HrpGdependent but HrpB-independent genes (RSc2285, RSc2286, RSc3101, RSp0883) and three genes of unknown function (RSp0817, RSp0818, RSp0819) (Table 1). It thus appears that a large majority (23 out of 30) of the genes repressed by PhcA in complete medium but PhcA-activated in planta belong to the HrpG-HrpB regulon which represent a set of key genes required for $R$. solanacearum pathogenesis [27]. Because the HrpB-HrpG regulon contains more than a hundred of genes [25,27], the relatively low number identified in our comparative analysis is probably due to the under-representation of expressed genes in the in planta transcriptomic study [7]. Genes controlled by HrpG and HrpB therefore appear to be subjected to a specific expression pattern, being activated by PhcA in planta as essential actors of the pathogenicity program (e.g T3SS) but repressed in replete (nutrient rich) conditions such as during

Table 1

List of genes up-regulated in complete medium and down-regulated in planta in a R. solanacearum strain GMI1000 phcA mutant.

\begin{tabular}{|c|c|c|c|c|c|c|}
\hline \multirow[b]{2}{*}{ Gene ID } & \multirow[b]{2}{*}{ Gene Name } & \multirow[b]{2}{*}{ Description } & \multicolumn{2}{|c|}{ CM } & \multicolumn{2}{|c|}{$\mathrm{PLT}^{(\mathrm{a})}$} \\
\hline & & & $\begin{array}{c}\text { log2 Fold } \\
\text { Change }\end{array}$ & padj,FDR & $\begin{array}{c}\text { log2 Fold } \\
\text { Change }\end{array}$ & padj,FDR \\
\hline RSc0616 & & CONSERVED HYPOTHETICAL PROTEIN & 2.37 & $8.50 \mathrm{E}-07$ & -3.26 & $2.79 \mathrm{E}-04$ \\
\hline RSc0617 & & SIGNAL PEPTIDE, CONSERVED HYPOTHETICAL PROTEIN & 1.71 & $3.86 \mathrm{E}-06$ & -2.63 & $8.46 \mathrm{E}-04$ \\
\hline RSc1729 & & TRANSCRIPTION REGULATOR & 2.07 & 2.14E-05 & -3.74 & $3.91 \mathrm{E}-03$ \\
\hline RSc2285 & & SIGNAL PEPTIDE, CONSERVED HYPOTHETICAL PROTEIN & 1.96 & 3.30E-06 & -3.73 & $1.72 \mathrm{E}-05$ \\
\hline RSc2286 & & CONSERVED HYPOTHETICAL PROTEIN & 1.02 & $1.31 \mathrm{E}-03$ & -3.94 & $1.75 \mathrm{E}-04$ \\
\hline RSc2510 & & SIGNAL PEPTIDE COG1638, TRAP-type C4-dicarboxylate transport system, periplasmic component & 2.21 & $1.09 \mathrm{E}-04$ & -2.10 & $3.32 \mathrm{E}-04$ \\
\hline RSc3101 & & SERINE PROTEASE & 2.86 & $1.39 \mathrm{E}-41$ & -2.72 & $3.64 \mathrm{E}-05$ \\
\hline RSp0200 & & OXIDOREDUCTASE PROTEIN WITH COG5322, Predicted dehydrogenase AND COG4992, Ornithine/acetylornithine aminotransferase DOMAINS & 1.23 & $6.22 \mathrm{E}-04$ & -3.96 & $6.38 \mathrm{E}-05$ \\
\hline RSp0201 & & CONSERVED HYPOTHETICAL PROTEIN & 1.18 & 2.45E-05 & -2.49 & $3.76 \mathrm{E}-04$ \\
\hline RSp0693 & hdfA & HDF BIOSYNTHESIS, COG0346, Lactoylglutathione lyase and related lyases & 4.85 & 1.58E-11 & -3.09 & 1.69E-03 \\
\hline RSp0694 & hdfB & HDF BIOSYNTHESIS, TRYPTOPHAN-2,3-DIOXYGENASE & 3.84 & 4.76E-17 & -3.05 & 3.80E-04 \\
\hline RSp0698 & hdfF & HDF BIOSYNTHESIS, AMINOTRANSFERASE & 2.91 & $2.81 \mathrm{E}-10$ & -2.46 & $1.41 \mathrm{E}-03$ \\
\hline RSp0817 & & OUTER MEMBRANE DRUG EFFLUX LIPOPROTEIN & 1.64 & 1.63E-04 & -2.29 & $2.17 \mathrm{E}-03$ \\
\hline RSp0818 & & TRANSMEMBRANE DRUG EFFLUX PROTEIN & 3.04 & $8.11 \mathrm{E}-41$ & -1.93 & $2.77 \mathrm{E}-04$ \\
\hline RSp0819 & & DRUG EFFLUX LIPOPROTEIN & 5.29 & $2.02 E-42$ & -1.93 & 3.37E-03 \\
\hline RSp0840 & & TRANSMEMBRANE METHYL-ACCEPTING CHEMOTAXIS TRANSDUCER & 5.78 & $1.25 \mathrm{E}-65$ & -2.63 & $1.02 \mathrm{E}-03$ \\
\hline RSp0855 & hrpY & TYPE 3 SECRETION SYSTEM PILUS SUBUNIT HRPY & 2.68 & 7.87E-08 & -1.26 & $1.85 \mathrm{E}-03$ \\
\hline RSp0873 & hrpB & TYPE 3 SECRETION SYSTEM REGULATORY PROTEIN HRPB & 3.92 & $2.39 \mathrm{E}-27$ & -2.23 & $3.88 \mathrm{E}-03$ \\
\hline RSp0874 & hrcC & TYPE 3 SECRETION SYSTEM CONSERVED PROTEIN HRCC & 4.16 & $6.29 \mathrm{E}-21$ & -2.00 & $2.83 \mathrm{E}-03$ \\
\hline RSp0875 & ripAC & TYPE 3 EFFECTOR PROTEIN RIPAC & 1.29 & 2.97E-03 & -1.20 & $2.64 \mathrm{E}-03$ \\
\hline RSp0876 & ripAB & TYPE 3 EFFECTOR PROTEIN RIPAB & 4.53 & $1.41 \mathrm{E}-41$ & -1.32 & 4.68E-04 \\
\hline RSp0877 & ripX & TYPE 3 EFFECTOR PROTEIN RIPX & 5.51 & 3.96E-32 & -1.20 & 8.83E-04 \\
\hline RSp0882 & & HYPOTHETICAL PROTEIN (HrPB regulon) & 3.69 & 1.47E-07 & -2.83 & $1.09 \mathrm{E}-03$ \\
\hline RSp0883 & & HYPOTHETICAL PROTEIN & 4.83 & $5.73 \mathrm{E}-30$ & -3.45 & $1.38 \mathrm{E}-03$ \\
\hline RSp0958 & & COG2846, Regulator of cell morphogenesis and NO signaling & 1.51 & $9.62 \mathrm{E}-04$ & -1.72 & $1.01 \mathrm{E}-03$ \\
\hline RSp1416 & & TRANSMEMBRANE,CONSERVED HYPOTHETICAL PROTEIN & 3.23 & 4.73E-17 & -4.27 & $3.92 \mathrm{E}-05$ \\
\hline RSp1461 & & SIGNAL PEPTIDE,CONSERVED HYPOTHETICAL PROTEIN & 4.55 & $6.08 \mathrm{E}-66$ & -2.20 & $2.94 \mathrm{E}-03$ \\
\hline RSp1555 & ripF1_1 & TYPE 3 SECRETION SYSTEM TRANSLOCON PROTEIN RIPF1_1 & 2.16 & $9.86 \mathrm{E}-10$ & -1.68 & $1.28 \mathrm{E}-04$ \\
\hline RSp1601 & ripAD & TYPE 3 EFFECTOR PROTEIN RIPAD & 2.50 & $1.45 \mathrm{E}-21$ & -2.43 & $8.56 \mathrm{E}-04$ \\
\hline RSp1652 & & COG1409, Phosphohydrolase & 1.61 & $2.97 \mathrm{E}-03$ & -3.88 & $6.56 \mathrm{E}-04$ \\
\hline
\end{tabular}

In blue are highlighted the hrpB-regulated genes; in grey the hrpG-regulated genes. CM (complete medium); PLT (in planta); padj,FDR (p-value adjusted FDR); (a) RNAseq data from Khokhani et al. [7]. Genes highlighted in red correspond to the down-regulated genes in a phcA mutant whereas the up-regulated genes are highlighted in green. 
Table 2

List of genes down-regulated in complete medium and up-regulated in planta in a $R$. solanacearum strain GMI1000 phcA mutant.

\begin{tabular}{|c|c|c|c|c|c|c|}
\hline \multirow[b]{2}{*}{ Gene ID } & \multirow[b]{2}{*}{ Gene Name } & \multirow[b]{2}{*}{ Description } & \multicolumn{2}{|c|}{ CM } & \multicolumn{2}{|c|}{$\mathrm{PLT}^{(\mathrm{a})}$} \\
\hline & & & $\begin{array}{l}\text { log2 Fold } \\
\text { Change }\end{array}$ & padj,FDR & $\begin{array}{l}\text { log2 Fold } \\
\text { Change }\end{array}$ & padj,FDR \\
\hline RSc0474 & & OXIDOREDUCTASE 3-HYDROXYACYL-COA DEHYDROGENASE & -1.11 & $6.85 \mathrm{E}-10$ & 1.43 & $1.38 \mathrm{E}-04$ \\
\hline RSc0481 & & ABC TRANSPORTER AMINO-ACID-BINDING PERIPLASMIC PROTEIN (PBP) & -1.38 & $1.10 \mathrm{E}-03$ & 1.63 & 2.41E-04 \\
\hline RSc0482 & gltJ & ABC TRANSPORTER GLUTAMATE/ASPARTATE TRANSMEMBRANE PROTEIN & -1.05 & 8.57E-04 & 1.32 & 3.04E-04 \\
\hline RSc0484 & gltL & ABC TRANSPORTER GLUTAMATE/ASPARTATE TRANSPORT ATP-BINDING PROTEIN & -1.27 & $1.28 \mathrm{E}-03$ & 1.68 & 3.61E-05 \\
\hline RSc0547 & & TRANSMEMBRANE SUGAR TRANSPORTER & -2.29 & 8.45E-08 & 2.51 & $1.51 \mathrm{E}-05$ \\
\hline RSc1014 & & TRANSCRIPTION REGULATOR & -1.38 & $1.28 \mathrm{E}-04$ & 1.14 & 7.63E-04 \\
\hline RSc1293 & emrA & TRANSMEMBRANE MULTIDRUG RESISTANCE PROTEIN A (TRANSLOCASE) & -2.25 & $5.88 \mathrm{E}-17$ & 1.26 & 3.86E-03 \\
\hline RSc1342 & ssuF & ORGANOSULFONATE UTILIZATION PROTEIN, MOLYBDOPTERIN-BINDING PROTEIN & -1.46 & $4.66 \mathrm{E}-15$ & 1.20 & 3.04E-03 \\
\hline RSc1380 & & ABC TRANSPORTER SUBSTATE-BINDING PERIPLASMIC PROTEIN (PBP) & -1.39 & $8.36 \mathrm{E}-07$ & 1.89 & 1.36E-04 \\
\hline RSc1381 & & ABC TRANSPORTER TRANSMEMBRANE PROTEIN & -1.47 & 3.49E-07 & 1.72 & $2.64 \mathrm{E}-03$ \\
\hline RSc1766 & & NADP-DEPENDENT OXIDOREDUCTASE & -1.02 & $6.00 \mathrm{E}-10$ & 1.07 & 3.21E-03 \\
\hline RSc1972 & phaP3 & POLYHYDROXYBUTYRATE GRANULE-ASSOCIATED PROTEIN (PHASIN) PHAP3 & -1.67 & 8.99E-11 & 2.07 & $5.46 \mathrm{E}-05$ \\
\hline RSc2353 & & SIGNAL PEPTIDE, CONSERVED HYPOTHETICAL PROTEIN & -1.39 & 1.13E-03 & 1.32 & $2.68 \mathrm{E}-03$ \\
\hline RSc2938 & & ABC TRANSPORTER TRANSMEMBRANE PROTEIN & -1.42 & 1.74E-06 & 4.39 & 3.15E-09 \\
\hline RSc3047 & & ABC TRANSPORTER ATP-BINDING PROTEIN & -1.27 & 3.33E-14 & 2.10 & 1.63E-04 \\
\hline RSc3048 & & ABC TRANSPORTER SUGAR ABC TRANSPORTER, PERMEASE PROTEIN & -1.92 & $8.58 \mathrm{E}-11$ & 1.63 & 2.13E-03 \\
\hline RSc3051 & & ABC TRANSPORTER COG1653, ABC-type sugar transport system, periplasmic component & -2.30 & $4.92 \mathrm{E}-11$ & 2.29 & 4.86E-06 \\
\hline RSc3128 & exac & OXIDOREDUCTASE NAD+ DEPENDENT ACETALDEHYDE DEHYDROGENASE & -1.34 & $1.72 \mathrm{E}-07$ & 1.77 & $6.64 \mathrm{E}-05$ \\
\hline RSc3270 & & TRANSMEMBRANE, CONSERVED HYPOTHETICAL PROTEIN & -2.03 & $5.81 \mathrm{E}-15$ & 1.58 & 4.73E-03 \\
\hline RSc3341 & & ABC TRANSPORTER TRANSMEMBRANE PROTEIN & -1.84 & 2.03E-07 & 2.26 & 3.17E-05 \\
\hline RSc3342 & & ABC TRANSPORTER SUBSTRATE-BINDING PERIPLASMIC PROTEIN (PBP) & -2.13 & 3.76E-09 & 2.26 & 6.67E-06 \\
\hline RSc3344 & & ABC TRANSPORTER ATP-BINDING PROTEIN & -1.07 & 3.40E-06 & 2.04 & 3.86E-05 \\
\hline RSp0053 & fdhA & OXIDOREDUCTASE GLUTATHIONE-INDEPENDENT FORMALDEHYDE DEHYDROGENASE & -1.16 & $1.82 \mathrm{E}-04$ & 1.96 & 7.89E-04 \\
\hline RSp1113 & & TRANSMEMBRANE MULTIDRUG-EFFLUX SYSTEM LIPOPROTEIN & -4.17 & 1.07E-129 & 1.32 & 9.14E-04 \\
\hline RSp1590 & & OXIDOREDUCTASE COG0665, Glycine/D-amino acid oxidases (deaminating) & -2.05 & $5.84 \mathrm{E}-11$ & 1.32 & 1.24E-03 \\
\hline RSp1591 & & OXIDOREDUCTASE ALDEHYDE DEHYDROGENASE & -2.13 & 4.38E-14 & 1.58 & $1.28 \mathrm{E}-03$ \\
\hline RSp1592 & & ABC TRANSPORTER ABC-type branched-chain amino acid transport systems, periplasmic component & -2.06 & $5.30 \mathrm{E}-14$ & 1.85 & $5.71 \mathrm{E}-05$ \\
\hline
\end{tabular}

CM (complete medium); PLT (in planta ); padj,FDR (p-value adjusted FDR); (a) RNAseq data from Khokhani et al. [7]. Genes highlighted in red correspond to the down-regulated genes in a phcA mutant whereas the up-regulated genes are highlighted in green.

the late stages of infection when the plant dies (Fig. 1). Expression of $R$. solanacearum T3SS genes is still effective when bacteria colonize xylem tissues $[28,29]$ and results of transcriptomic analyses indicate that PhcA plays a key role in the fine-tuning of T3SS expression. These results also implies that a specific signal is perceived in plant xylem tissues by bacteria in order to relieve the PhcA-dependent repression that is exerted on $h r p G / h r p B$, and/or to specifically convert PhcA from a repressor to an activator of this class of genes through an unknown mechanism. It remains to be determined whether this signal is independent of the plant cell contact activation pathway known to activate HrpG/ HrpB [30].

\section{Conclusion}

PhcA is a central regulatory node in RSSC strains, which controls a broad array of biological functions with a transcriptional control extending up to $30 \%$ of the bacterial genome. As such, PhcA controls other functions beside pathogenicity stricto sensu which participate to the global cell homeostasis (metabolism, energy storage). The comparison of the transcriptomic profiles of $R$. pseudosolanacearum grown in complete medium in vitro or after in planta growth revealed that a large majority of the PhcA controlled genes followed the same regulation pattern in both conditions, except for a set of HrpG-HrpB regulated genes including the T3SS and T3Es. This latter class of genes appears to be specifically induced by PhcA in the plant environment whereas this regulator represses their expression in complete medium. The large set of direct and indirect targets identified in this study will contribute to enrich our knowledge of the intricate regulatory network coordinating the expression of virulence and metabolic functions in this model plant pathogen [31].

\section{Funding}

This work was supported by the 'Institut National de la Recherche Agronomique' (INRA) (Plant Health Division grant AAP SPE 2015) and the French Laboratory of Excellence project TULIP (ANR-10-LABX-41; ANR-11-IDEX-0002-02). Rémi Peyraud was supported by EMBO (Long-
Term Fellowship ALTF 1627-2011) and Marie Curie Actions (EMBOCOFUND2010, GA-2010-267146). Anthony Perrier was supported by INRA, Plant Health Division (SPE) and the 'Région Occitanie' (No. 15000183).

\section{Conflicts of interest}

The authors report no conflict of interest.

\section{Appendix A. Supplementary data}

Supplementary data related to this article can be found at http://dx. doi.org/10.1016/j.micpath.2018.01.028.

\section{References}

[1] M.A. Schell, Control of virulence and pathogenicity genes of Ralstonia solanacearum by an elaborate sensory network, Annu. Rev. Phytopathol. 38 (2000) 263-292, http://dx.doi.org/10.1146/annurev.phyto.38.1.263.

[2] S. Genin, T.P. Denny, Pathogenomics of the Ralstonia solanacearum species complex, Annu. Rev. Phytopathol. 50 (2012) 67-89, http://dx.doi.org/10.1146/annurevphyto-081211-173000.

[3] S. Genin, B. Brito, T.P. Denny, C. Boucher, Control of the Ralstonia solanacearum Type III secretion system (Hrp) genes by the global virulence regulator PhcA, FEBS Lett. 579 (2005) 2077-2081, http://dx.doi.org/10.1016/j.febslet.2005.02.058.

[4] K. Kai, H. Ohnishi, M. Shimatani, S. Ishikawa, Y. Mori, A. Kiba, K. Ohnishi, M. Tabuchi, Y. Hikichi, Methyl 3-hydroxymyristate, a diffusible signal mediating phc quorum sensing in Ralstonia solanacearum, Chembiochem Eur. J. Chem. Biol. 16 (2015) 2309-2318, http://dx.doi.org/10.1002/cbic.201500456.

[5] S.J. Clough, K.E. Lee, M.A. Schell, T.P. Denny, A two-component system in Ralstonia (Pseudomonas) solanacearum modulates production of PhcA-regulated virulence factors in response to 3-hydroxypalmitic acid methyl ester, J. Bacteriol. 179 (1997) 3639-3648.

[6] R. Peyraud, L. Cottret, L. Marmiesse, J. Gouzy, S. Genin, A resource allocation tradeoff between virulence and proliferation drives metabolic versatility in the plant pathogen Ralstonia solanacearum, PLoS Pathog. 12 (2016) e1005939, , http://dx doi.org/10.1371/journal.ppat.1005939.

[7] D. Khokhani, T.M. Lowe-Power, T.M. Tran, C. Allen, A single regulator mediates strategic switching between attachment/spread and growth/virulence in the plant pathogen Ralstonia solanacearum, mBio 8 (2017) e00895-17, , http://dx.doi.org/ 10.1128/mBio.00895-17.

[8] Y. Mori, S. Ishikawa, H. Ohnishi, M. Shimatani, Y. Morikawa, K. Hayashi, K. Ohnishi, A. Kiba, K. Kai, Y. Hikichi, Involvement of ralfuranones in the quorum 
sensing signalling pathway and virulence of Ralstonia solanacearum strain OE1-1, Mol. Plant Pathol. (2017), http://dx.doi.org/10.1111/mpp.12537 n/a-n/a

[9] M. Salanoubat, S. Genin, F. Artiguenave, J. Gouzy, S. Mangenot, M. Arlat, A. Billault, P. Brottier, J.C. Camus, L. Cattolico, M. Chandler, N. Choisne, C. Claudel-Renard, S. Cunnac, N. Demange, C. Gaspin, M. Lavie, A. Moisan, C. Robert, W. Saurin, T. Schiex, P. Siguier, P. Thébault, M. Whalen, P. Wincker, M. Levy, J. Weissenbach, C.A. Boucher, Genome sequence of the plant pathogen Ralstonia solanacearum, Nature 415 (2002) 497-502, http://dx.doi.org/10.1038/ 415497a.

[10] L. Plener, P. Manfredi, M. Valls, S. Genin, PrhG, a transcriptional regulator responding to growth conditions, is involved in the control of the type III secretion system regulon in Ralstonia solanacearum, J. Bacteriol. 192 (2010) 1011-1019, http://dx.doi.org/10.1128/JB.01189-09.

[11] A. Perrier, R. Peyraud, D. Rengel, X. Barlet, E. Lucasson, J. Gouzy, N. Peeters, S. Genin, A. Guidot, Enhanced in planta fitness through adaptive mutations in EfpR, a dual regulator of virulence and metabolic functions in the plant pathogen Ralstonia solanacearum, PLoS Pathog. 12 (2016) e1006044, , http://dx.doi.org/10. 1371/journal.ppat.1006044.

[12] S. Anders, W. Huber, Differential expression analysis for sequence count data, Genome Biol. 11 (2010) R106, , http://dx.doi.org/10.1186/gb-2010-11-10-r106.

[13] K. Strimmer, A unified approach to false discovery rate estimation, BMC Bioinf. 9 (2008) 303, http://dx.doi.org/10.1186/1471-2105-9-303.

[14] C.K. Wairuri, J.E. van der Waals, A. van Schalkwyk, J. Theron, Ralstonia solanacearum needs Flp pili for virulence on potato, Mol. Plant-Microbe Interact. MPMI 25 (2012) 546-556, http://dx.doi.org/10.1094/MPMI-06-11-0166.

[15] E. Titarenko, E. López-Solanilla, F. García-Olmedo, P. Rodríguez-Palenzuela, Mutants of Ralstonia (Pseudomonas) solanacearum sensitive to antimicrobial peptides are altered in their lipopolysaccharide structure and are avirulent in tobacco, J. Bacteriol. 179 (1997) 6699-6704.

[16] M. Pötter, H. Müller, F. Reinecke, R. Wieczorek, F. Fricke, B. Bowien, B. Friedrich, A. Steinbüchel, The complex structure of polyhydroxybutyrate (PHB) granules: four orthologous and paralogous phasins occur in Ralstonia eutropha, Microbiol. Read. Engl 150 (2004) 2301-2311, http://dx.doi.org/10.1099/mic.0.26970-0.

[17] Y. Murai, S. Mori, H. Konno, Y. Hikichi, K. Kai, Ralstonins a and B, lipopeptides with chlamydospore-inducing and phytotoxic activities from the plant pathogen Ralstonia solanacearum, Org. Lett. 19 (2017) 4175-4178, http://dx.doi.org/10. 1021/acs.orglett.7b01685.

[18] F. Baldeweg, H. Kage, S. Schieferdecker, C. Allen, D. Hoffmeister, M. Nett, Structure of ralsolamycin, the interkingdom morphogen from the crop plant pathogen Ralstonia solanacearum, Org. Lett. 19 (2017) 4868-4871, http://dx.doi.org/10. 1021/acs.orglett.7b02329.

[19] D. Hernández-Romero, F. Solano, A. Sanchez-Amat, Polyphenol oxidase activity expression in Ralstonia solanacearum, Appl. Environ. Microbiol. 71 (2005) 6808-6815, http://dx.doi.org/10.1128/AEM.71.11.6808-6815.2005.

[20] J. Tans-Kersten, D. Brown, C. Allen, Swimming motility, a virulence trait of
Ralstonia solanacearum, is regulated by FlhDC and the plant host environment, Mol. Plant-Microbe Interact. MPMI 17 (2004) 686-695, http://dx.doi.org/10.1094/ MPMI.2004.17.6.686.

[21] H. Liu, Y. Kang, S. Genin, M.A. Schell, T.P. Denny, Twitching motility of Ralstonia solanacearum requires a type IV pilus system, Microbiol. Read. Engl 147 (2001) 3215-3229.

[22] J. Huang, B.F. Carney, T.P. Denny, A.K. Weissinger, M.A. Schell, A complex network regulates expression of eps and other virulence genes of Pseudomonas solanacearum, J. Bacteriol. 177 (1995) 1259-1267.

[23] T. Yoshimochi, Y. Hikichi, A. Kiba, K. Ohnishi, The global virulence regulator PhcA negatively controls the Ralstonia solanacearum hrp regulatory cascade by repressing expression of the PrhIR signaling proteins, J. Bacteriol. 191 (2009) 3424-3428, http://dx.doi.org/10.1128/JB.01113-08.

[24] S. Cunnac, A. Occhialini, P. Barberis, C. Boucher, S. Genin, Inventory and functional analysis of the large Hrp regulon in Ralstonia solanacearum: identification of novel effector proteins translocated to plant host cells through the type III secretion system, Mol. Microbiol. 53 (2004) 115-128, http://dx.doi.org/10.1111/j.13652958.2004.04118.x.

[25] A. Occhialini, S. Cunnac, N. Reymond, S. Genin, C. Boucher, Genome-wide analysis of gene expression in Ralstonia solanacearum reveals that the hrpB gene acts as a regulatory switch controlling multiple virulence pathways, Mol. Plant-Microbe Interact. MPMI 18 (2005) 938-949, http://dx.doi.org/10.1094/MPMI-18-0938.

[26] F. Delaspre, C.G. Nieto Peñalver, O. Saurel, P. Kiefer, E. Gras, A. Milon, C. Boucher, S. Genin, J.A. Vorholt, The Ralstonia solanacearum pathogenicity regulator HrpB induces 3-hydroxy-oxindole synthesis, Proc. Natl. Acad. Sci. U. S. A 104 (2007) 15870-15875, http://dx.doi.org/10.1073/pnas.0700782104.

[27] M. Valls, S. Genin, C. Boucher, Integrated regulation of the type III secretion system and other virulence determinants in Ralstonia solanacearum, PLoS Pathog. 2 (2006) e82, , http://dx.doi.org/10.1371/journal.ppat.0020082.

[28] F. Monteiro, S. Genin, I. van Dijk, M. Valls, A luminescent reporter evidences active expression of Ralstonia solanacearum type III secretion system genes throughout plant infection, Microbiology 158 (2012) 2107-2116, http://dx.doi.org/10.1099/ mic.0.058610-0.

[29] J.M. Jacobs, L. Babujee, F. Meng, A. Milling, C. Allen, The in planta transcriptome of Ralstonia solanacearum: conserved physiological and virulence strategies during bacterial wilt of tomato, mBio 3 (2012) e00114-12, , http://dx.doi.org/10.1128/ mBio.00114-12.

[30] D. Aldon, B. Brito, C. Boucher, S. Genin, A bacterial sensor of plant cell contact controls the transcriptional induction of Ralstonia solanacearum pathogenicity genes, EMBO J. 19 (2000) 2304-2314, http://dx.doi.org/10.1093/emboj/19.10. 2304.

[31] R. Peyraud, L. Cottret, L. Marmiesse, S. Genin, Control of primary metabolism by a virulence regulatory network promotes robustness in a plant pathogen, Nat. Commun. 9 (1) (2018) 418, http://dx.doi.org/10.1038/s41467-017-02660-4. 